\title{
Management of Stem Rot of Groundnut caused by Sclerotium rolfsii Sacc. with Actinomycetes
}

\author{
G. Leona*, R. Sudhakar, G. Uma Devi and T. Uma Maheswari \\ Department of Plant Pathology, College of Agriculture, Professor Jayashankar Telangana \\ State Agricultural University, Rajendranagar, Hyderabad-030, Telangana, India \\ *Corresponding author
}

\section{A B S T R A C T}

\section{Keywords}

Groundnut, stem rot, Sclerotium rolfsii, biological control, actinomycetes

\section{Article Info}

Accepted: 04 November 2020 Available Online: 10 December 2020
Sclerotium rolfsii Sacc. is an destructive soil borne fungal pathogen with wide host range that includes groundnut, an important oil seed crop in India. Biological control offers an interesting alternative to fungicides for sustainable management of soil borne diseases. A total of ten isolates of actinomycetes were collected from different districts of Telangana and tested for their inhibitory activity against Sclerotium rolfsii Sacc., the stem rot of pathogen. These isolates were identified as Streptomyces spp. on the basis of standard bacteriological test and 16S RNA gene sequence analysis. Among the various isolates tested in vitro, three isolates (RGP-49\%, NDG- 47.7\%, YLD-45\% inhibition) were found effective in inhibiting the mycelial growth of Sclerotium rolfsii in dual culture assay. These three potential isolates had shown $100 \%$ compatibility with the systemic fungicides (carbendazim, tebuconazole, propiconazole and azoxystrobin) whereas less compatibility with the non-systemic fungicides (captan and mancozeb) when tested by disc diffusion method. Seeds of groundnut when treated with Streptomyces spp. increased root length, shoot length and seedling vigour. Greenhouse studies revealed that talc-based formulations of Streptomyces spp. increased the germination percentage by RGP- 89\%, NDG- 85\%, YLD-84\%. Among the three potential isolates maximum disease control was obtained when seed treatment and soil application of isolate RGP (Streptomyces parvulus) and YLD (Streptomyces werraensis) was used in controlling stem rot under greenhouse conditions.

\section{Introduction}

Groundnut (Arachis hypogaea L.) is an important oil seed crop in the world belonging to the Leguminosae family. It is one of the essential food and cash crops of our country and is considered as the 'king' of oilseeds. It is also known as wonder nut, peanuts or monkey nuts and provides over 30 essential nutrients. It is a valuable source of protein, niacin, fiber, vitamin $\mathrm{E}$ and anti-oxidants. The seeds are valued for high 40-48 per cent edible oil 
content, 22-26 per cent digestible protein content, 26 per cent carbohydrate, 3 per cent fat and high calcium, thiamine and niacin contents, which make a substantial contribution of protein for human and animal nutrition. They can be consumed directly or processed into oil meal or cake which serves as an important source of protein for livestock or further processed into confectionery products or snack food. The oil cake is rich in nitrogen, potassium, phosphorous and can be used as fertilizers. Immense health benefits are due to the presence of antioxidants and resveratrol. The groundnuts are also used in the manufacturing of soaps, shaving creams, cosmetics and lubricants.

Groundnut is cultivated worldwide in an area of $28 \mathrm{M}$ ha with a total production of $47 \mathrm{Mt}$ averaging a productivity of $1.6 \mathrm{t} \mathrm{ha}^{-1}$ (FAO, 2017). The leading producers of the groundnut crop include China (54\%), India (22\%), and USA $(9.03 \%)$. In India, the crop is mostly grown in the states of Gujarat, Andhra Pradesh, Telangana, Tamil Nadu, Karnataka, Rajasthan and Maharashtra constituting about 80 percent of the total area and production of groundnut.

In India a large number of diseases attack groundnut (Mayee and Datar, 1988). Among many soil borne diseases, stem rot or white mold caused by Sclerotium rolfsii Sacc. (Saccardo, 1911) is an important disease causing significant yield losses in several groundnut growing countries (Mehan et al., 1994). The pathogen attacks host plant during at all stages when conditions are favourable (Punja, 1985) and yield losses over 25\% have been reported (Mayee and Datar, 1988).

The pathogen survives as resistant structures called sclerotia in the soil which serves as primary source of inoculum for disease in the absence of susceptible host and remain viable for a long period (Backman and Brenneman,
1997; Aycock, 1966). High temperature of $30^{\circ} \mathrm{C}$, dense planting, frequent irrigation, moist conditions favours infection and fungal mycelial spread within and between plants (Aycock, 1966; Paolo, 1933; Punja, 1985).

The stem rot infected plant produces typical symptoms which include dark brown lesions on the stem just below the soil surface followed by drooping and wilting of entire plant (Garren, 1959; Backman and Brenneman, 1997). Such wilted plants show cottony mycelial growth of the fungus, girdling of basal part of the stem, spreading in both directions covering the stem and roots followed by formation of light to dark brown sclerotia (Mehan et al., 1995). Whitish mycelial growth with sclerotial bodies resembling mustard seeds can be observed at collar region. Infected seeds appear to be bluish-black, reducing its economic value (Garren and Higgins, 1947, Subrahmanyam et al., 2012).

Sclerotium rolfsii causes immediate knock down effect on plant by producing organic acids like oxalic acid and enzymes like cellulases (Bateman, 1970; Bateman, 1972; Bateman and Beer, 1965) Hence, yield losses caused by stem rot are directly proportional to the disease incidence. Further, once established in the soil, the soil turns sick, and hence management of the disease is very difficult.

Different practices are recommended for management of groundnut stem rot such as deep summer ploughing, destruction of plant debris, crop rotation with jowar and bajra, seed treatment with carbendazim or captan or mancozeb or tebuconazole, soil drenching with hexaconazole, application of ammonium sulphate or calcium ammonium nitrate instead of urea and application of gypsum at flowering stage. Management of diseases caused by these soil borne plant pathogenic 
fungi by chemicals are not practicable owing to high cost besides causing environmental pollution and resistance development in target fungus (Biswas and Sen, 2000; Pant and Mukopadhyay, 2001; Patibanda et al., 2002; Uma Maheshwari et al., 2002; Rudresh et al., 2005). Further, no single treatment is full proof and disease continues to cause losses in farmers' fields.

At this juncture, biological control seems to offer a practicable approach as it has several advantages (when applied either alone or in combination with other management practices) like eco-friendliness, effective against soil borne disease, growth promoting activity etc., which cannot be possible by chemicals.

Actinobacteria are excellent choices as plant disease control agents due to their ability to produce fungicidally important compounds that antagonize many phytopathogens (Xiao et al., 2002; Meschke et al., 2012).

These are a diverse group of free living saprobic mycelial bacteria present abundantly in the soil, maintaining the structure and integrity of soil. They also take part in the recycling of soil nutrients. However, reports on the role of actinomycetes as biocontrol agents is meagre. Keeping this in view the objectives formulated were:

To isolate and identify actinomycetes from the rhizosphere soil of groundnut crop.

To screen the actinomycete isolates against Sclerotium rolfsii under in vitro conditions.

To study the compatibility of actinomycetes isolates with commonly used fungicides.

To evaluate the potential isolates of actinomycetes against Sclerotium rolfsii causing stem rot of groundnut in glass house.

\section{Materials and Methods}

\section{Isolation of pathogen and actinomycetes}

The fungus Sclerotium rolfsii, was isolated from a stem rot infected plants collected from the groundnut fields of Seed Technology Research Centre (SRTC), Rajendranagar, Hyderabad and maintained on potato dextrose agar (PDA) plates incubated at $25^{\circ} \mathrm{C}$ in a BOD incubator for five days. The pathogenicity of the fungal culture was proved by using Koch's postulates. After 14 days sclerotia were harvested from plates and stored at $4^{\circ} \mathrm{C}$ for further studies.

Actinomycetes were isolated from rhizosphere soils of groundnut collected from different districts of Telangana state (Table 1). Soil samples were suspended in sterile water $(10 \mathrm{gm}$ in $100 \mathrm{ml})$ and agitated for $45 \mathrm{~min}$ at $160 \mathrm{rpm}$.

The supernatants were serially diluted upto $10^{-}$

6 and spreaded $(0.1 \mathrm{ml})$ evenly on the actinomycetes isolation agar medium incubated at $28 \pm 2{ }^{\circ} \mathrm{C}$ for 15 days. Subculturing was done on starch casein agar medium to get pure colonies. These isolates were identified as Streptomyces spp. on the basis of standard bacteriological test and 16S RNA gene sequence analysis.

\section{Phenotypic Characterization of Actinomycetes}

After the incubation period the plates were examined for typical actinomycetes colonies such as round, small, opaque, compact, frequently pigmented (white, brown, gray pink or other colors) and appear dull looking (Praveen et al., 2017). The phenotypic features such as aerial mass colour, colony shape, colony surface, colony colour, spore formation of actinomycetes isolates was examined on starch ceasin medium. 


\section{Biochemical} Actinomycetes

\section{Characterization}

of

Biochemical characterization of actinomycetes isolates were studied to understand the basic physiology of the soil actinomycetes. Biochemical tests analysis such as oxidase test, catalase test, voges prausker's test, methyl red test, indole test, gelatin liquefaction, hydrolysis of startch and hydrogen sulphide test were done ((Holt, 1989).

\section{Molecular Actinomycetes}

\section{Characterization}

The molecular characterization of pure cultures of actinomycetes was done at ICRISAT. Sequenced DNA data were complied and analyzed with a Basic local alignment search tool (BLAST) (www.ncbi.nlm.nih.gov). The genomic DNA sequence was used for species identification and 16S rDNA phylogenetic tree was built by Neighbor-Joining method constructed with MUSCLE and the evolutionary analyses were conducted in MEGA X.

In vitro screening of actinomycetes against S. rolfsii

Ten isolates of actinomycetes were tested for antifungal activity against $S$. rolfsii under in vitro conditions using a dual culture assay (Anjaiah et al., 2003 and Adhilakshmi et al., 2013). Fresh cultures of actinomycetes were streaked on one end of the starch casein agar media plates and incubated for $96 \mathrm{~h}$ at $28 \pm 2$ ${ }^{\circ} \mathrm{C}$. After incubation period, a mycelial plug (5 $\mathrm{mm}$ diameter) from a 3-days-old $S$. rolfsii culture was placed on the opposite side, perpendicular to the actinomycetes in the media plates. Control plates were maintained with $S$. rolfsii mycelial discs alone. The Petri dishes were incubated for 7 days at $28 \pm 2{ }^{\circ} \mathrm{C}$, until the fungus completely covered the control plates. Three replications were maintained for each isolate. Per cent inhibition (I \%) of the pathogen by the antagonistic isolates was calculated with the formula (Dennis and Webster 1971). Per cent inhibition $(\mathrm{I} \%)=(\mathrm{C}-\mathrm{T}) / \mathrm{C} \times 100$, where, $\mathrm{I}=$ Per cent reduction in growth of the pathogen, $\mathrm{C}=$ Radial growth of pathogen in control, $\mathrm{T}=$ Radial growth of pathogen in treatment

\section{Preparation of talc based formulation of potential Streptomyces spp.}

Talc-based formulations of effective of Streptomyces spp. (RGP, NDG and YLD) were prepared on carrier material, talcum powder. One hundred grams of carrier material was taken and the $\mathrm{pH}$ was adjusted to 7 by adding $\mathrm{CaCO}_{3}$ at the rate of $15 \mathrm{~g} / \mathrm{kg}$. Carboxy methyl cellulose (CMC) was then added at the rate of $10 \mathrm{~g} / \mathrm{kg}$ and mixed well. The mixtures was then autoclaved for $30 \mathrm{~min}$ at $121^{\circ} \mathrm{C}\left(15 \mathrm{lb} / \mathrm{inch}^{2}\right)$. After autoclaving, 400 $\mathrm{ml}$ of bacterial suspension $\left(1 \times 10^{8} \mathrm{cfu} / \mathrm{ml}\right)$ was added to the sterilized carrier material $(1 \mathrm{~kg})$ and thorough mixed followed by drying aseptically and then grounded to powder. They were then packed in sterile polythene bags and stored at $4{ }^{\circ} \mathrm{C}$ (Vidhyasekaran and Muthamilan,1995). The concentration of colony-forming units was obtained using the formula: Number of $\mathrm{cfu} / \mathrm{g}=$ Number of colonies / (Amount of sample plated $\mathrm{x}$ Dilution) X 100

\section{Efficacy of Streptomyces spp. on plant growth}

The influence of Streptomyces spp. (RGP, NDG and YLD) on germinability and vigor of peanut seedlings was evaluated using the Ragdoll method (Chamblee and Green 1995) with slight modifications. K-6 variety seeds were surface sterilized using $2.5 \%$ sodium hypochlorite solution for $2 \mathrm{~min}$ and washed several times with distilled water. Seed treatments (ST) were prepared by treating surface sterilized seeds with talc formulation 
of Streptomyces spp. Treated peanut seeds were placed on germination towels and incubated in growth chambers at $28{ }^{\circ} \mathrm{C}$ for 15 days. Four replications were kept for each treatment, with 10 seeds per replication.

Seeds soaked in sterile distilled water served as control. Seven days after incubation, number of germinated seeds was recorded (Sai et al., 2018). At the end of 15 days, root and shoot lengths of seedlings was recorded and the seedling vigour index was calculated as per the following formula: Vigour index $=$ germination per cent $\mathrm{X}$ (root length + shoot length)

\section{Compatibility of actinomycetes with fungicides in vitro}

Streptomyces spp. isolates were tested in vivo for their compatibility with the fungicides by Disc diffusion method. A loop full of actively growing culture of Streptomyces spp. isolates was added into starch casein broth and incubated at $28 \pm 2^{\circ} \mathrm{C}$ for 3 days at $180 \mathrm{r} \mathrm{min}^{-}$ 1. After incubation period, $0.1 \mathrm{ml}$ of Streptomyces spp. isolate suspension was uniformly spreaded over the starch casein agar media plates. Fungicide solutions of required concentrations i.e. 50, 100, 250 and 500 ppm, a.i. for systemic fungicides (propiconazole, tebuconazole, azoxystrobin, carbendazin) and 1000, 1500, 2000 and 2500 ppm a.i. for nonsystemic fungicides (captan and mancozeb) were prepared in separated test tube. $5 \mathrm{~mm}$ diameter sterile filter paper discs were dipped in fungicide solutions at required concentrations and placed on the starch casein agar. Discs dipped in normal water and placed on the starch casein agar served as control. For each treatment three replications were maintained and incubated at $28 \pm 2{ }^{\circ} \mathrm{C}$. After 14 days Per cent inhibition of Streptomyces spp. with certain concentration was recorded with the formula (Louis et al., 2016): Per cent inhibition $(\mathrm{I} \%)=(\mathrm{C}-\mathrm{T}) / \mathrm{C} \times 100$, where, $\mathrm{I}=$ Per cent reduction in growth of the
Streptomyces spp., $\mathrm{C}=$ Radial growth of Streptomyces spp. in control, $\mathrm{T}=$ Radial growth of Streptomyces spp. in treatment

Biological control assay with Streptomyces spp. formulation

Pot culture studies were conducted to test the antagonists for their efficacy in vivo. Two different application methods i.e., seed treatment and soil application were used to assess the biocontrol potential in decreasing stem rot of groundnut caused by $S$. rolfsii (Adhilakshmi et al., 2013 and Jacob et al., 2018).

S. rolfsii, mass multiplied on sorghum grains was mixed with sandy loam soil @ $4 \mathrm{~g} \mathrm{~kg}^{-1}$ soil in the pots and were incubated for twenty four hours before sowing.

Groundnut seeds of K-6 variety were treated with talc based formulation of potential Streptomyces spp.@10 g kg${ }^{-1}$ of seed in the infested soil.

For soil applications, the talc power formulations of potential Streptomyces spp. were added to FYM and neem cake mixture (in 2:90:8 ratio) and incubated for 15 days. Such antagonist fortified FYM was added to soil @ 2g/pot before sowing groundnut seeds on the top 2" layer. For treatments involving seed treatment with fungicide and biocontrol agent. First seeds were coated with fungicide followed by Streptomyces spp. talc powder and were sown twenty four hours after $S$. rolfsii inoculation.

Pathogen inoculated and uninoculated pots were maintained as checks and three replications were maintained for each treatment. Seeds were sown @ 5 per pot. Irrigation was done as and when necessary to maintain soil moisture. Data on number of seeds germinated and number of plants survived was recorded 30 days after sowing as 
per the formula: Per cent germination = Number of seeds germinated in each treatment / Total number of plants assessed X 100 and Per cent disease incidence $=$ Number of infected plants / Total number of plants assessed X 100

\section{Results and Discussion}

Biological control of stem rot of groundnut has been pursued by using antagonistic microorganism (Rakh et al., 2011, Jacob et al., 2016).

In the present investigation ten actinomycetes isolates were isolated, screened for their biocontrol potential against groundnut stem rot pathogen Sclerotium rolfsii in vitro and the selected potential isolates were further assessed for the compatibility with commonly used fungicides and in pot culture against groundnut stem rot.

Phenotypic Characterization of Actinomycetes

Ten pure and clear distinguishable colonies were isolated from 10 soil samples, these strains were slow to moderate growing and showed colony colours like white and grey.

Binocular compound microscope (100X) studies were used to examine the spore surface and spore shape. (Table.1).

\section{Biochemical Characterization of Actinomycetes}

The biochemical characterization of the potential isolate, NDG and YLD included oxidase test, catalase test, gelatin liquefaction, hydrolysis of startch and hydrogen sulphide test (Table.2).

\section{Molecular Characterization of} Actinomycetes

16S rRNA gene sequencing established revealed that the nine isolates belong to the genus Streptomyces except isolate LKP which was identified as Nocardiopsis of Phylum Actinobacteria (Table.3). The $16 \mathrm{~S}$ rRNA gene sequences were submitted to the Gen Bank with the accession numbers.

Table.1 Collection of isolates of actinomycetes from the rhizosphere of groundnut plants from different districts of Telangana

\begin{tabular}{|c|c|c|c|c|}
\hline Village & District & Latitude & Longitude & $\begin{array}{c}\text { Isolate } \\
\text { code }\end{array}$ \\
\hline Kadarigudem & Warangal & 17.812097 & 79.591928 & KDG \\
\hline Yellanda & Warangal & 17.790303 & 79.593930 & YLD \\
\hline Dharmapur & Warangal & 17.589325 & 79.395619 & DMP \\
\hline Ramojikummarigudem & Warangal & 17.772540 & 79.575977 & RKG \\
\hline Palem & Mahabubnagar & 16.555308 & 78.209277 & PLM \\
\hline Nandigama & Mahabubnagar & 18.049111 & 79.826284 & NDG \\
\hline Dasaripally & Mahabubnagar & 17.990992 & 79.860546 & DSP \\
\hline Rangapur & Ranga Reddy & 17.544298 & 79.522845 & RGP \\
\hline Lenkallapally & Nalagonda & 18.027734 & 79.856665 & LKP \\
\hline Kamapally & Nizamabad & 17.986614 & 79.858700 & KMP \\
\hline
\end{tabular}


Table.2 Phenotypic characterization of different isolates of actinomycetes

\begin{tabular}{|c|c|c|c|c|c|c|c|c|c|c|c|c|}
\hline S.no & Strain & $\begin{array}{c}\text { Aerial } \\
\text { mass } \\
\text { colour }\end{array}$ & $\begin{array}{l}\text { Colour of } \\
\text { substrate } \\
\text { mycelium }\end{array}$ & $\begin{array}{c}\text { Melanoid } \\
\text { pigment }\end{array}$ & $\begin{array}{c}\text { Diffusible } \\
\text { pigment }\end{array}$ & $\begin{array}{l}\text { Colony } \\
\text { Shape }\end{array}$ & $\begin{array}{l}\text { Colony } \\
\text { Surface }\end{array}$ & $\begin{array}{l}\text { Colony } \\
\text { colour }\end{array}$ & $\begin{array}{c}\text { Light } \\
\text { microscopy }\end{array}$ & $\begin{array}{c}\text { Spore } \\
\text { surface } \\
\text { morphology }\end{array}$ & $\begin{array}{l}\text { Spore } \\
\text { shape }\end{array}$ & $\begin{array}{c}\text { Speed of } \\
\text { growth }\end{array}$ \\
\hline 1 & KDG & White(W) & $\begin{array}{l}\text { Moderate } \\
\text { yellow }\end{array}$ & None & None & Round & Smooth & White & Flexible(F) & Smooth & $\begin{array}{c}\text { Rod } \\
\text { shaped }\end{array}$ & Moderate \\
\hline 2 & RGP & White(W) & $\begin{array}{c}\text { Greyish } \\
\text { brown }\end{array}$ & Brown & $\begin{array}{l}\text { Pale } \\
\text { Brown }\end{array}$ & Irregular & Rough & White & $\operatorname{Spira}(\mathrm{S})$ & Smooth & $\begin{array}{c}\text { Rod } \\
\text { shaped }\end{array}$ & Slow \\
\hline 3 & YLD & White(W) & Red & None & None & Round & Powdery & White & $\operatorname{Spira}(\mathrm{S})$ & Smooth & $\begin{array}{c}\text { Rod } \\
\text { shaped }\end{array}$ & Moderate \\
\hline 4 & DMP & White(W) & $\begin{array}{l}\text { Greyish } \\
\text { yellow }\end{array}$ & Brown & None & $\begin{array}{l}\text { Round } \\
\text { with } \\
\text { lines }\end{array}$ & Rough & White & $\operatorname{Spira}(\mathrm{S})$ & Smooth & $\begin{array}{c}\text { Rod } \\
\text { shaped }\end{array}$ & Good \\
\hline 5 & PLM & White(W) & $\begin{array}{l}\text { Bright } \\
\text { yellow }\end{array}$ & None & None & $\begin{array}{l}\text { Round } \\
\text { radiated }\end{array}$ & Smooth & White & $\operatorname{Spira}(\mathrm{S})$ & Smooth & $\begin{array}{c}\text { Rod } \\
\text { shaped }\end{array}$ & Good \\
\hline 6 & LKP & White(W) & $\begin{array}{l}\text { Light } \\
\text { Yellow }\end{array}$ & None & None & $\begin{array}{l}\text { Round } \\
\text { with } \\
\text { feathers }\end{array}$ & Smooth & White & $\operatorname{Spira}(\mathrm{S})$ & Smooth & $\begin{array}{c}\text { Rod } \\
\text { shaped }\end{array}$ & Good \\
\hline 7 & NDG & White(W) & $\begin{array}{l}\text { Brownish } \\
\text { yellow }\end{array}$ & Brown & None & $\begin{array}{l}\text { Button } \\
\text { with } \\
\text { feathers }\end{array}$ & Powdery & White & Spira(S) & Smooth & $\begin{array}{c}\text { Rod } \\
\text { shaped }\end{array}$ & Good \\
\hline 8 & RKG & White & $\begin{array}{l}\text { Pale } \\
\text { yellow }\end{array}$ & None & None & $\begin{array}{c}\text { Double } \\
\text { ring }\end{array}$ & Powdery & White & $\operatorname{Spira}(\mathrm{S})$ & Smooth & $\begin{array}{l}\text { Ovoid } \\
\text { shaped }\end{array}$ & Good \\
\hline 9 & DSP & Gray & $\begin{array}{l}\text { Yellowish } \\
\text { gray }\end{array}$ & None & None & $\begin{array}{l}\text { Two } \\
\text { round } \\
\text { circles }\end{array}$ & Rough & $\begin{array}{l}\text { White and } \\
\text { grey }\end{array}$ & $\begin{array}{l}\text { Retinaculum } \\
\text { Apertum(RA) }\end{array}$ & Smooth & $\begin{array}{c}\text { Rod } \\
\text { shaped }\end{array}$ & Good \\
\hline 10 & KMP & $\begin{array}{l}\text { Light } \\
\text { gray }\end{array}$ & $\begin{array}{l}\text { Light } \\
\text { gray }\end{array}$ & None & None & Round & Smooth & $\begin{array}{l}\text { White and } \\
\text { grey }\end{array}$ & $\operatorname{Spira}(\mathrm{S})$ & Smooth & $\begin{array}{c}\text { Rod } \\
\text { shaped }\end{array}$ & Good \\
\hline
\end{tabular}


Table.3 Biochemical characteristics of different isolates of actinomycetes

\begin{tabular}{|c|c|c|c|c|c|c|c|c|c|c|}
\hline Isolate & $\begin{array}{l}\text { Gram's } \\
\text { staining }\end{array}$ & $\begin{array}{c}\text { Oxidase } \\
\text { test }\end{array}$ & $\begin{array}{c}\text { Catalase } \\
\text { test }\end{array}$ & $\begin{array}{c}\text { Voges } \\
\text { Prausker's } \\
\text { test }\end{array}$ & $\begin{array}{l}\text { Methyl } \\
\text { red test }\end{array}$ & $\begin{array}{l}\text { Indole } \\
\text { test }\end{array}$ & $\begin{array}{c}\text { Gelatin } \\
\text { liquefaction }\end{array}$ & $\begin{array}{l}\text { Hydrolysis } \\
\text { of starch }\end{array}$ & $\begin{array}{l}\text { Hydrogen } \\
\text { sulphide } \\
\text { production }\end{array}$ & $\begin{array}{l}\text { Coagulation } \\
\text { of milk }\end{array}$ \\
\hline KDG & + & + & + & - & + & - & - & + & - & - \\
\hline RGP & + & + & + & - & - & - & + & + & + & + \\
\hline YLD & + & + & + & - & + & - & + & + & + & + \\
\hline DMP & + & + & + & - & + & - & + & + & + & + \\
\hline PLM & + & + & + & - & + & - & - & + & + & - \\
\hline LKP & + & + & + & - & + & - & + & + & - & + \\
\hline NDG & + & + & + & - & - & - & + & + & + & + \\
\hline RKG & + & + & + & - & + & - & + & + & + & + \\
\hline DSP & + & + & + & - & + & - & + & + & + & + \\
\hline KMP & + & + & + & - & - & - & + & + & + & + \\
\hline
\end{tabular}

(+) Presence / Positive
(-) Absence / Negative

Table.4 Molecular characterization of actinomycetes isolates

\begin{tabular}{|c|c|c|c|}
\hline Isolate & $\begin{array}{c}\text { Identification of the actinomycete through 16S } \\
\text { rRNA gene sequencing }\end{array}$ & Type strain & $\begin{array}{c}\text { Sequence } \\
\text { similarity }\end{array}$ \\
\hline DSP & Streptomyces griseostramineus & NBRC 12781 & MW070527 \\
\hline LKP & Nocardiopsis synnemataformans & DSM 44143 & MW070526 \\
\hline NDG & Streptomyces werraensis & NRBC 13404 & MW070529 \\
\hline KDG & Streptomyces aureofaciens & DSM 40127 & MW070532 \\
\hline PLM & Streptomyces albaduncus & JCM 4715 & MW070531 \\
\hline RGP & Streptomyces parvulus & NBRC 13193 & MW073390 \\
\hline YLD & Streptomyces aurantiacus & NBRC 13017 & MW073391 \\
\hline KMP & Streptomyces cacaoi & DSM 40057 & MW070530 \\
\hline DPM & Streptomyces griesus & DSM 40226 & MW073392 \\
\hline RKG & Streptomyces lusitanus & 97.01 \\
\hline
\end{tabular}


Table.5 Effect of different isolates of Streptomyces on radial growth of S. rolfsii in dual culture assay

\begin{tabular}{|c|c|c|c|c|c|c|c|c|}
\hline \multirow[b]{2}{*}{ Isolate } & \multicolumn{4}{|c|}{ Radial growth of Sclerotium rolfsii } & \multirow[b]{2}{*}{ Day 6} & \multirow{2}{*}{$\begin{array}{c}\text { Inhibition } \\
(\%)\end{array}$} & \multirow{2}{*}{$\begin{array}{c}\text { Day } 8 \\
\text { Radial } \\
\text { growth }(\mathrm{cm})\end{array}$} & \multirow{2}{*}{$\begin{array}{c}\text { Inhibition } \\
(\%)\end{array}$} \\
\hline & Day 2 & & Day 4 & & & & & \\
\hline KDG & 1.36 & 10.82 & 3.23 & 3.03 & 5.33 & 4.20 & 7.20 & 0 \\
\hline RGP & 1.13 & 26.09 & 2.03 & 39.00 & 3.03 & 45.51 & 3.66 & 49.06 \\
\hline YLD & 1.13 & 26.09 & 2.43 & 27.00 & 3.73 & 32.94 & 3.96 & 44.90 \\
\hline PLM & 1.36 & 10.82 & 3.03 & 9.00 & 5.53 & 0.61 & 7.20 & 0.00 \\
\hline LKP & 1.13 & 26.09 & 2.63 & 21.00 & 5.36 & 3.59 & 7.20 & 0.00 \\
\hline NDG & 1.23 & 19.56 & 2.23 & 33.00 & 3.03 & 45.51 & 3.76 & 47.75 \\
\hline RKG & 1.23 & 19.56 & 3.23 & 3.03 & 5.41 & 2.40 & 7.20 & 0.00 \\
\hline DSP & 1.20 & 21.72 & 3.06 & 7.98 & 5.36 & 3.59 & 7.20 & 0.00 \\
\hline KMP & 1.23 & 19.56 & 3.13 & 6.06 & 5.53 & 0.61 & 7.20 & 0.00 \\
\hline
\end{tabular}

Note: Data are the mean of three replications 
Table.6 Compatibility of different concentrations of fungicides with isolate RGP, YLD and NDG of Streptomyces spp.

\begin{tabular}{|c|c|c|c|c|c|c|}
\hline \multirow{3}{*}{$\begin{array}{c}\text { Fungicide } \\
\text { A= Systemic } \\
\text { B= Non-systemic }\end{array}$} & \multirow[t]{3}{*}{ Isolate } & \multicolumn{4}{|c|}{$\begin{array}{l}\text { Per cent inhibition of Streptomyces with fungicide } \\
\text { Concentration }\end{array}$} & \multirow[t]{3}{*}{$\begin{array}{c}\text { Mean bioagent YLD } \\
\text { inhibition }\end{array}$} \\
\hline & & $\mathrm{A}=50 \mathrm{ppm}$ & $\mathrm{A}=100$ ppm & $\mathrm{A}=250$ ppm & $\mathrm{A}=\mathbf{5 0 0} \mathbf{p p m}$ & \\
\hline & & $B=1000 \mathrm{ppm}$ & $\mathrm{B}=1500 \mathrm{ppm}$ & $B=2000 \mathrm{ppm}$ & $B=2500 \mathrm{ppm}$ & \\
\hline Captan (B) & $\begin{array}{l}\text { RGP } \\
\text { YLD } \\
\text { NDG }\end{array}$ & $\begin{array}{l}0(0.0) \\
0(0.0) \\
0(0.0)\end{array}$ & $\begin{array}{l}0(0.0) \\
0(0.0) \\
0(0.0)\end{array}$ & $\begin{array}{c}0.833(5.234) \\
0(0.0) \\
0.767(4.908)\end{array}$ & $\begin{array}{c}0.933(5.540) \\
0(0.0) \\
0.9(5.36)\end{array}$ & $\begin{array}{c}0.442(2.693) \\
0(0.0) \\
0.408(2.586)\end{array}$ \\
\hline Mancozeb (B) & $\begin{array}{l}\text { RGP } \\
\text { YLD } \\
\text { NDG }\end{array}$ & $\begin{array}{l}0(0.0) \\
0(0.0) \\
0(0.0)\end{array}$ & $\begin{array}{l}0(0.0) \\
0(0.0) \\
0(0.0)\end{array}$ & $\begin{array}{l}0.867(5.338) \\
0.667(4.679) \\
0.767(5.019)\end{array}$ & $\begin{array}{c}1.0(5.732) \\
0.833(5.234) \\
0.933(5.540)\end{array}$ & $\begin{array}{l}0.467(2.767) \\
0.375(2.478) \\
0.425(2.640)\end{array}$ \\
\hline $\begin{array}{l}\text { Carbendazim } \\
\text { (A) }\end{array}$ & $\begin{array}{l}\text { RGP } \\
\text { YLD } \\
\text { NDG }\end{array}$ & $\begin{array}{l}0(0.0) \\
0(0.0) \\
0(0.0)\end{array}$ & $\begin{array}{l}0(0.0) \\
0(0.0) \\
0(0.0)\end{array}$ & $\begin{array}{l}0(0.0) \\
0(0.0) \\
0(0.0)\end{array}$ & $\begin{array}{l}0(0.0) \\
0(0.0) \\
0(0.0)\end{array}$ & $\begin{array}{l}0(0.0) \\
0(0.0) \\
0(0.0)\end{array}$ \\
\hline $\begin{array}{l}\text { Propiconazole } \\
\text { (A) }\end{array}$ & $\begin{array}{l}\text { RGP } \\
\text { YLD } \\
\text { NDG }\end{array}$ & $\begin{array}{l}0(0.0) \\
0(0.0) \\
0(0.0)\end{array}$ & $\begin{array}{l}0(0.0) \\
0(0.0) \\
0(0.0)\end{array}$ & $\begin{array}{l}0(0.0) \\
0(0.0) \\
0(0.0)\end{array}$ & $\begin{array}{l}0(0.0) \\
0(0.0) \\
0(0.0)\end{array}$ & $\begin{array}{l}0(0.0) \\
0(0.0) \\
0(0.0)\end{array}$ \\
\hline $\begin{array}{l}\text { Tebuconazole } \\
\text { (A) }\end{array}$ & $\begin{array}{l}\text { RGP } \\
\text { YLD } \\
\text { NDG }\end{array}$ & $\begin{array}{l}0(0.0) \\
0(0.0) \\
0(0.0)\end{array}$ & $\begin{array}{l}0(0.0) \\
0(0.0) \\
0(0.0)\end{array}$ & $\begin{array}{l}0(0.0) \\
0(0.0) \\
0(0.0)\end{array}$ & $\begin{array}{l}0(0.0) \\
0(0.0) \\
0(0.0)\end{array}$ & $\begin{array}{l}0(0.0) \\
0(0.0) \\
0(0.0)\end{array}$ \\
\hline $\begin{array}{l}\text { Azoxystrobin } \\
\text { (A) }\end{array}$ & $\begin{array}{l}\text { RGP } \\
\text { YLD } \\
\text { NDG }\end{array}$ & $\begin{array}{l}0(0.0) \\
0(0.0) \\
0(0.0)\end{array}$ & $\begin{array}{l}0(0.0) \\
0(0.0) \\
0(0.0)\end{array}$ & $\begin{array}{l}0(0.0) \\
0(0.0) \\
0(0.0)\end{array}$ & $\begin{array}{l}0(0.0) \\
0(0.0) \\
0(0.0)\end{array}$ & $\begin{array}{l}0(0.0) \\
0(0.0) \\
0(0.0)\end{array}$ \\
\hline $\begin{array}{l}\text { Concentration } \\
\text { mean }(\%)\end{array}$ & $\begin{array}{l}\text { RGP } \\
\text { YLD } \\
\text { NDG }\end{array}$ & $\begin{array}{l}0(0.0) \\
0(0.0) \\
0(0.0)\end{array}$ & $\begin{array}{l}0(0.0) \\
0(0.0) \\
0(0.0)\end{array}$ & $\begin{array}{l}0.283(1.762) \\
0.111(0.779) \\
0.250(1.654)\end{array}$ & $\begin{array}{l}0.322(1.879) \\
0.138(0.872) \\
0.306(1.829)\end{array}$ & - \\
\hline Factors & & Fungicide (F) & Concentration(C) & F X C & & \\
\hline C.D. & $\begin{array}{l}\text { RGP } \\
\text { YLD } \\
\text { NDG }\end{array}$ & $\begin{array}{l}0.024(0.071) \\
0.014(0.046) \\
0.024(0.074)\end{array}$ & $\begin{array}{l}0.019(0.058) \\
0.011(0.038) \\
0.019(0.006)\end{array}$ & $\begin{array}{l}0.048(0.141) \\
0.027(0.092) \\
0.048(0.148)\end{array}$ & & \\
\hline $\operatorname{SE}(d)$ & $\begin{array}{l}\text { RGP } \\
\text { YLD } \\
\text { NDG }\end{array}$ & $\begin{array}{l}0.012(0.035) \\
0.007(0.028) \\
0.012(0.037)\end{array}$ & $\begin{array}{c}0.01(0.029) \\
0.006(0.019) \\
0.010(0.030)\end{array}$ & $\begin{array}{l}0.024(0.020) \\
0.014(0.046) \\
0.024(0.074)\end{array}$ & & \\
\hline SE(m) & $\begin{array}{l}\text { RGP } \\
\text { YLD } \\
\text { NDG }\end{array}$ & $\begin{array}{l}0.008(0.141) \\
0.005(0.016) \\
0.008(0.016)\end{array}$ & $\begin{array}{l}0.007(0.070) \\
0.004(0.013) \\
0.007(0.013)\end{array}$ & $\begin{array}{l}0.017(0.050) \\
0.010(0.030) \\
0.017(0.052)\end{array}$ & & \\
\hline
\end{tabular}

Notes: Data are mean of three replications.

Figures in parentheses are arc sine transformed values. 
Table.7 Efficacy of Streptomyces spp. on plant growth

\begin{tabular}{|c|c|c|c|c|}
\hline $\begin{array}{c}\text { Streptomyces sp. } \\
\text { strains }\end{array}$ & Germination $(\%)$ & Shoot length $(\mathbf{c m})$ & Root length $(\mathbf{c m})$ & Vigour index \\
\hline RGP & $\begin{array}{c}99 \\
(84.30)\end{array}$ & 14.6 & 25.833 & $4,003.73$ \\
\hline YLD & $\begin{array}{c}97 \\
(80.06)\end{array}$ & 13.667 & 23.167 & $3,573.00$ \\
\hline NDG & 97.66 & 13.9 & 23.833 & $3,685.13$ \\
\hline Control & $(81.24)$ & 12.333 & 19 & $3,008.83$ \\
\hline C.D. & 96 & 1.142 & 1.851 & 283.811 \\
\hline SE(m) & $178.50)$ & 0.345 & 0.559 & 85.698 \\
\hline SE(d) & 0.527 & 0.488 & 0.791 & 121.195 \\
\hline C.V. & 0.745 & 4.383 & 4.217 & 4.16 \\
\hline
\end{tabular}

Notes: Data are mean of three replications.

Figures in parentheses are arc sine transformed values.

Plate.1 Dual culture technique employed with isolates of actinomycetes against $S$. rolfsii
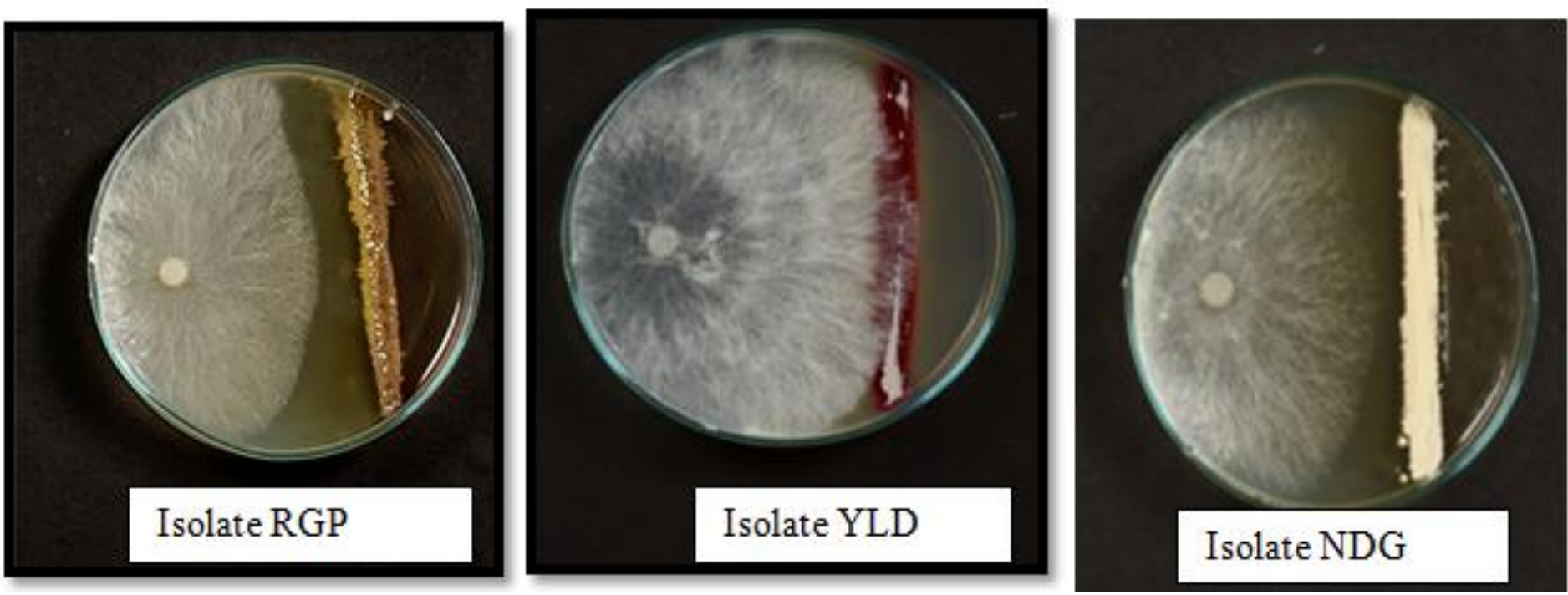
Table.8 Evaluation of biocontrol potential of isolates of Streptomyces spp. against S. rolfsii in vivo on per cent germination and disease control

\begin{tabular}{|c|c|c|c|c|}
\hline Treatment & Treatments & $\underset{\%}{\text { Germination }}$ & $\begin{array}{c}\text { Disease } \\
\text { incidence \% }\end{array}$ & $\begin{array}{c}\text { Disease } \\
\text { control \% }\end{array}$ \\
\hline T1 & $\begin{array}{c}\text { Seed treatment (ST) with isolate RGP of } \\
\text { Streptomyces spp. }\end{array}$ & $\begin{array}{c}80 \\
(63.42)\end{array}$ & $\begin{array}{c}14 \\
(21.93)\end{array}$ & $\begin{array}{c}86 \\
(68.03)\end{array}$ \\
\hline T2 & $\begin{array}{c}\text { Soil application (SA) of isolate RGP of Streptomyces } \\
\text { spp. }\end{array}$ & $\begin{array}{c}78 \\
(62.007)\end{array}$ & $\begin{array}{l}14.167 \\
(22.09)\end{array}$ & $\begin{array}{l}85.833 \\
(67.86)\end{array}$ \\
\hline $\mathbf{T 3}$ & $\begin{array}{c}\text { Seed treatment }+ \text { Soil application }(\mathrm{ST}+\mathrm{SA}) \text { of isolate } \\
\text { RGP of Streptomyces spp. }\end{array}$ & $\begin{array}{c}89 \\
(70.61)\end{array}$ & $\begin{array}{c}0 \\
(0.0)\end{array}$ & $\begin{array}{c}100 \\
(90.00)\end{array}$ \\
\hline T4 & $\begin{array}{l}\text { Seed treatment of Carbendazim @ 0.1\% + ST with } \\
\text { isolate RGP of Streptomyces spp. }\end{array}$ & $\begin{array}{c}80 \\
(63.42)\end{array}$ & $\begin{array}{c}14 \\
(21.95)\end{array}$ & $\begin{array}{c}86 \\
(68.00)\end{array}$ \\
\hline T5 & $\begin{array}{l}\text { Seed treatment (ST) with isolate YLD of } \\
\text { Streptomyces spp. }\end{array}$ & $\begin{array}{c}77.66 \\
(61.78)\end{array}$ & $\begin{array}{c}20 \\
(26.55)\end{array}$ & $\begin{array}{c}80 \\
(63.41)\end{array}$ \\
\hline T6 & $\begin{array}{l}\text { Soil application (SA) with isolate YLD of } \\
\text { Streptomyces spp. }\end{array}$ & $\begin{array}{c}75.5 \\
(60.308)\end{array}$ & $\begin{array}{l}20.333 \\
(26.78)\end{array}$ & $\begin{array}{l}79.667 \\
(63.18)\end{array}$ \\
\hline T7 & $\begin{array}{c}\text { Seed treatment }+ \text { Soil application (ST+SA) of isolate } \\
\text { YLD of Streptomyces spp. }\end{array}$ & $\begin{array}{c}84 \\
(66.42)\end{array}$ & $\begin{array}{c}4 \\
(11.51)\end{array}$ & $\begin{array}{c}96 \\
(78.44)\end{array}$ \\
\hline T8 & $\begin{array}{l}\text { Seed treatment of Carbendazim @ 0.1\%)+ ST with } \\
\text { isolate YLD of Streptomyces spp. }\end{array}$ & $\begin{array}{l}76.333 \\
(60.87)\end{array}$ & $\begin{array}{l}20.667 \\
(24.01)\end{array}$ & $\begin{array}{l}79.333 \\
(62.94)\end{array}$ \\
\hline T9 & $\begin{array}{c}\text { Seed treatment (ST) with isolate NDG of } \\
\text { Streptomyces spp. }\end{array}$ & $\begin{array}{l}77.667 \\
(61.77)\end{array}$ & $\begin{array}{c}16 \\
(23.56)\end{array}$ & $\begin{array}{c}84 \\
(66.40)\end{array}$ \\
\hline T10 & $\begin{array}{c}\text { Soil application (SA) with isolate NDG of } \\
\text { Streptomyces spp. }\end{array}$ & $\begin{array}{c}76 \\
(60.64)\end{array}$ & $\begin{array}{c}16.3 \\
(23.79)\end{array}$ & $\begin{array}{c}83.7 \\
(66.16)\end{array}$ \\
\hline T11 & $\begin{array}{c}\text { Seed treatment + Soil application (ST+SA) of isolate } \\
\text { NDG of Streptomyces spp. }\end{array}$ & $\begin{array}{l}84.667 \\
(66.93)\end{array}$ & $\begin{array}{c}0 \\
(0.0)\end{array}$ & $\begin{array}{c}100 \\
(90.00)\end{array}$ \\
\hline T12 & $\begin{array}{c}\text { Seed treatment of Carbendazim @ 0.1\% + ST with } \\
\text { isolate NDG of Streptomyces spp. }\end{array}$ & $\begin{array}{l}76.667 \\
(61.09)\end{array}$ & $\begin{array}{l}16.333 \\
(23.82)\end{array}$ & $\begin{array}{l}83.667 \\
(66.13)\end{array}$ \\
\hline T13 & Seed treatment of Carbendazim @ 0.1\% & $\begin{array}{l}66.667 \\
(54.71)\end{array}$ & $\begin{array}{c}45.42 \\
(42.35)\end{array}$ & $\begin{array}{c}54.58 \\
(47.13)\end{array}$ \\
\hline T14 & Pathogen inoculated control (Pathogen check) & $\begin{array}{l}52.667 \\
(44.71)\end{array}$ & $\begin{array}{l}80.333 \\
(63.66)\end{array}$ & $\begin{array}{l}19.667 \\
(26.30)\end{array}$ \\
\hline T15 & Un-inoculated control (Absolute check) & $\begin{array}{c}70 \\
(56.77)\end{array}$ & $\begin{array}{c}0 \\
(0.0)\end{array}$ & $\begin{array}{c}100 \\
(90.00)\end{array}$ \\
\hline & C.D. & 2.393 & 1.77 & 1.770 \\
\hline & $\mathrm{SE}(\mathrm{m})$ & 0.824 & 0.61 & 0.610 \\
\hline
\end{tabular}

Notes: Data are mean of three replications;

Figures in parentheses are arc sine transformed values.

In vitro screening of actinomycetes against S. rolfsii

Dual culture studies revealed that both the interacting fungi and the actinomycetes isolates could come together after eight days of inoculation. Among the 10 isolates, 3 isolates had shown inhibition aganist growth of $S$. rolfsii. Isolate RGP produced a zone of inhibition of 49.06 per cent whereas, isolate 
NDG and isolate YLD produced zone of inhibition of 47.75 per cent and 44.9 per cent indicating that secondary metabolites of Streptomyces spp. might be involved in inhibiting the growth of $S$. rolfsii (Table.4, Plate.1).

\section{Efficacy of Streptomyces spp. on plant growth}

Streptomyces spp. produce growth-promoting factors such as auxins and gibberellins which promote plant growth (Jacob et al., 2018). The study indicated that the potential isolates RGP, NDG and YLD of Streptomyces spp. significantly increased per cent seed germination, root length, shoot length and seedling vigour over control. Among the three potential isolates tested, the isolate RGP was the most effective in promoting growth of plant followed by NDG and YLD. Significant increase in vigour index was recorded in seeds treated with talc formulation of the isolate RGP (4003.73) followed by the isolate NDG (3685.13) and YLD (3573) than that of the untreated control (3008.83) (Table.5).

\section{Compatibility of actinomycetes with fungicides in vitro}

Among the 10 isolates, only 3 isolates had shown inhibitory effect against S.rolfsii i.e isolates RGP, NDG and YLD. Hence, these 3 isolates were used to check the combatibility of different commonly used fungicides. These isolates had shown 100 per cent compatibility with the systemic fungicides viz., carbendazim, tebuconazole, propiconazole and azoxystrobin at all concentrations whereas less compatibility was shown by non-systemic fungicides (captan and mancozeb). Thus the present study indicates the possibilities of combined use of systemic fungicides with bio control agent Streptomyces sp. for the effective management of stem rot of groundnut (Table.6).

\section{Green house experiment}

The freshly prepared antagonists bio agents must be used immediately hence, talc formulations were used for easy storage and proper handling for commercial use. The talc based formulations of three Streptomyces spp. isolates were used for their efficacy in controlling $S$. rolfsii under greenhouse conditions. Inoculation of $S$. rolfsii in groundnut had caused disease incidence upto 80 per cent under pot culture experiments. Pot culture studies revealed that seed treatment with Streptomyces isolate RGP, isolate NDG and isolate YLD prevented seed rot, seedling rot and collar rot caused by $S$. rolfsii in groundnut. All the treatments proved better in reducing stem rot of groundnut in comparison to pathogen inoculated check.

Seed treatment, soil application or combination of fungicide and biocontrol agent were found effective in increasing seed germination per cent.

But, higher seed germination results were obtained when both seed treatment and soil application of potential biocontrol agents were used $(\mathrm{ST}+\mathrm{SA})$ when compared with absolute check.

Disease control obtained when the isolate RGP was used as talc formulation is $86 \%$ followed by NDG (84\%) and YLD (80\%). But, the maximum disease control was obtained when combination of both seed treatment and soil application $(\mathrm{ST}+\mathrm{SA})$ of isolate RGP and NDG were used and had shown $100 \%$ disease control in green house studies.

No additional benefit could be derived by combination treatments of fungicide and biocontrol agent indicating that biocontrol agent can successfully be substituted for fungicidal treatment. Integration of chemical 
and biocontrol agents did not give any additional benefit in decreasing the groundnut stem rot with germination on par with absolute check (Table.7).

Results indicated that seed treatment or soil application of powder formulations of all the three strains of Streptomyces sp. effectively reduced the incidence of stem rot under greenhouse conditions. Among them, RGP (Streptomyces parvulus) and YLD (Streptomyces werraensis) were found highly effective in controlling stem rot under greenhouse conditions.

\section{Acknowledgement}

The authors are thankful to ICRISAT, Hyderabad.

\section{References}

Adhilakshmi, M., Latha, P., Paranidharana, V., Balachandar, D., Ganesamurthy, K and Velazhahana, R. 2013. Biological control of stem rot of groundnut (Arachis hypogae L.) caused by Sclerotium rolfsii Sacc. with actinomycetes. Archives of Phytopathology and Plant Protection. 47: 298-311.

Anjaiah, V., Cornelis, P., Koedam, N. 2003. Effect of genotype and root colonization in biological control of fusarium wilts in pigeonpea and chickpea by Pseudomonas aeruginosa PNA1. Canadian Journal of Microbiology. 49: 85-91.

Aycock, R., 1966. Stem rot and other diseases caused by Sclerotium rolfsii or the status of Rolfs' fungus after 70 years. Raleigh: North Carolina State University Technical Bulletin.174.

Backman, P. A., Brenneman, T. B. 1997. Stem rot, in: Compendium of Peanut Diseases Burelle, N.K., Porter, D.M., Kabana, R.R., Smith, D.H., Subrahmanyam, P., APS Press, St. Paul, Minn, USA, 2nd edition Eds. 36-37.

Bateman, D. F., 1970. Depletion of the galacturonic acid content in bean hypocotyl cell walls during pathogenesis by Rhizoctonia solaniand Sclerotium rolfsii.
Phytopathology. 60: 1846-1847.

Bateman, D. F., 1972. The polygalacturonase complex produced by Sclerotium rolfsii. Physiological Plant Pathology. 2: 175-184.

Bateman, D. F., Beer, S. V., 1965. Simultaneous production and synergistic action of oxalic acid and polygalacturonase during pathogenesis by Sclerotiorum rolfsii. Phytopathology. 55: 204-211.

Biswas, K. K and Sen, C. 2000. Management of stem rot of groundnut caused by Sclerotium rolfsii through Trichoderma harzianum. Indian Phytopathology. 53: 290-295.

Chamblee, D. S., Green, J. T. 1995. Production and utilization of pastures and forages in North Carolina. North Carolina Agricultural Research Service Technical Bulletin. 305153.

Dennis, C and Webster, J. 1971. Antagonistic properties of species groups of Trichoderma 1. Production of non-volatile antibiotics. Transactions of the British Mycological Society. 57: 25-39.

Garren and Higgins. 1947. Symptomology and management of crown rot (Sclerotium rolfsii) of french bean. Journal of Mycology and Plant Pathology, 34: 820-823.

Garren, K. H.1959. The stem rot of peanuts and its control. Technical Bulletein. 144. Virginia Agricultural Experiment Station. 29.

Holt, J. G. 1989. Bergey`s Manual of Systematic Bacteriology. Williams and Williams, Baltimore, MD.

Jacob, S., Rao, S R., Sudini, H K., 2018. Streptomyces sp. RP1A-12 mediated control of peanut stem rot caused by Sclerotium rolfsii Journal of Integrative Agriculture Volume 17, Issue 4, April 2018, Pages 892900.

Jacob, S., Rao, S R., Kumar, K V K., Varshney, R., Sudini, H K. 2016. Assessing the prospects of Streptomyces sp. RP1A-12 in managing groundnut stem rot disease caused by Sclerotium rolfsii Sacc. Journal of General Plant Pathology. 82(2): 96-104.

Louis, V. J., JiphyJose, P., and Pushpalatha. 2016. Compatibility of Pseudomonas fluorescens with Fungicides used in Banana Cultivation. International Journal of Agriculture Innovations and Research. 5(3): 2319-1473.

Mayee, C. D and Datar, V. V. 1988. Diseases of 
groundnut in the tropics. Review of Tropical Plant Pathology. 5: 169-198.

Mehan, V. K., Mayee, C. D., Brenneman, T.B. and MC Donald, D. 1995. Stem and pod rot of groundnut. ICAISAT, Information Bulletin No. 44. 19-27.

Mehan, V. K., Mayee, C. D and McDonald, D. 1994. Management of Sclerotium rolfsiicaused stem and pod rots of groundnut - a critical review. Internatioal Journal of Pest Management. 40: 313-320.

Meschke, H., Walter, S and Schrempf H. 2012. Characterization and localization of prodiginines from Streptomyces lividans suppressing Verticillium dahliae in the absence or presence of Arabidopsis thaliana. Environmental Microbiology. 14: 940-592.

Pant, R and Mukhopadhyay, A. N. 2001. Integrated Management of seed and seedling rot complex of soybean. Indian Phytopathology. 54: 346-350.

Paolo. 1933. Studies on sclerotial root rot disease of groundnut (Arachis hypogea L.) by Sclerotium rolfsii Sacc. Madras Agricultural Journal. 51: 367-368.

Patibanda, A. K., Upadhyay, J. P. Mukhopadhyay, A. N. 2002. Efficacy of Trichoderma harzianum Rifai alone or in combination with fungicides against Sclerotium wilt of groundnut. Journal of Biological Control. 16(1): 57-63.

Praveen Kumar, P., Sagaya Jansi, R., Saravana Kumar, $\mathrm{P}$ et al.,2017. Optimization of biosynthesis parameters, partial purification and characterization of extracellular lipase from soil derived Streptomyces sp. Loyola Lipase-1. Biocatalysis and Agricultural Biotechnology. 12: 241-247.

Punja, Z. K. 1985. The biology, ecology, and control of Sclerotium rolfsii. Annual Review of Phytopathology. 23: 97-127.
Rakh R R, Raut L S, Dalvi S M, Manwar A V. 2011. Biological control of Sclerotium rolfsii, causing stem rot of groundnut by Pseudomonas cf. monteilii 9. Recent Research in Science and Technilogy. 3:2634

Rudresh, D. L., Shivaprakash, M. K and Prasad, R. D. 2005. Potential of Trichoderma spp as biocontrol agents of pathogens involved in wilt complex (Cicer arietinum. L). Journal of Biological Control. 19(2): 157166.

Saccardo, P.A. 1911. Notae Mycological. Annuals of Mycology. 9: 247.

Sai, S. K. P. V., Davide, G and Emilio, S. 2018. Plant growth promoting and biocontrol activity of Streptomyces spp. as endophytes. International Journal of Molecular Sciences. 19(4) : 952.

Subrahmanyam, P., Wongkaew, S., Reddy, D. V. R., Demski, J. W., McDonald, D., Sharma, S.B., Smith, D.H., Nigam, S. N. and Sudini, H., 2012. Field diagnosis of groundnut diseases. Information Bulletin no. 36 (revised). (in En. Fr. Summaries in En, Fr, Es.) Patancheru, A.P. 502 324. India: International Crops Research Institute for the Semi-Arid Tropics. 88 pp. ISBN 92-9066255-7.

Uma Maheswari, M. P., Muthuswamy, $\mathrm{M}$ and Alice, D. 2002. Evaluation of antagonists against jasmine wilt caused by Sclerotium rolfsii (Sacc.). Journal of Biological Control. 16: 135-140.

Vidhyasekaran P, Muthamilan M. 1995. Development of formulations of Pseudomonas fluorescens for control of chickpea wilt. Plant Disease. 79: 782-786.

Xiao, K; Kinkel, L. L and Samac, D. A. 2002. Biological control of Phytophthora root rots on Alfalfa and Soybean with Streptomyces. Biological control. 23:285 - 295.

\section{How to cite this article:}

Leona, G., R. Sudhakar, G. Uma Devi and Uma Maheswari, T. 2021. Management of Stem Rot of Groundnut caused by Sclerotium rolfsii Sacc. with Actinomycetes. Int.J.Curr.Microbiol.App.Sci. 9(12): 3587-3601. doi: https://doi.org/10.20546/ijcmas.2020.912.427 